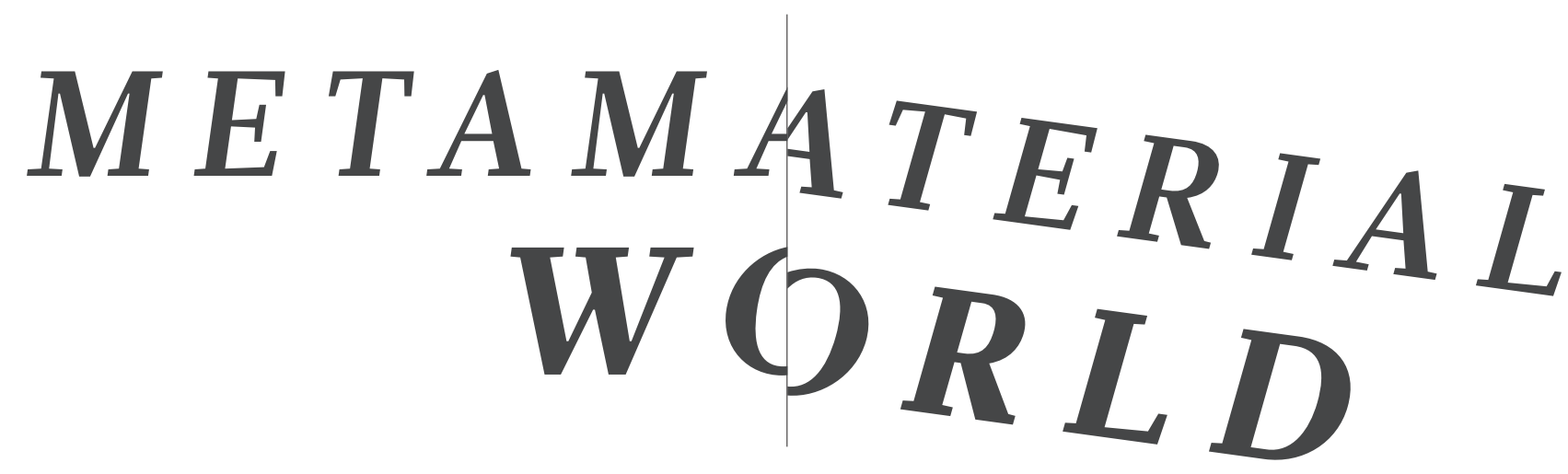

BY LEE BILLINGS

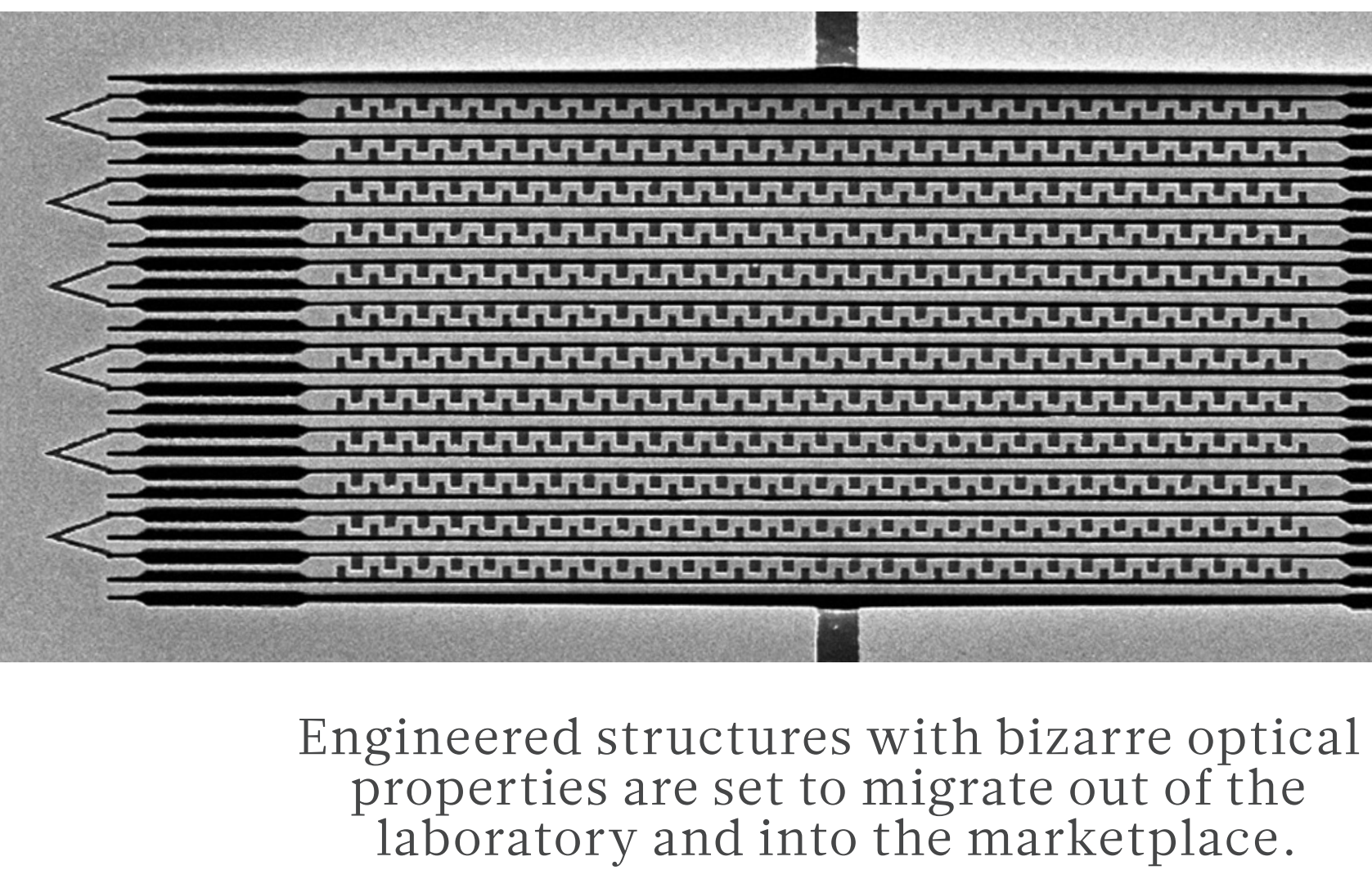

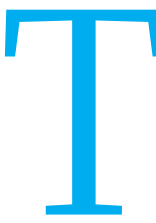

om Driscoll would be happy if he never heard the phrase 'Harry Potterstyle invisibility cloak' again. But he knows he will. The media can't seem to resist using it when they report the latest advances in metamaterials - arrays of minuscule 'elements' that bend, scatter, transmit or otherwise shape electromagnetic radiation in ways that no natural material can. It is true that metamaterials could, in principle, route light around objects and render them invisible, not unlike the cloak of a certain fictional wizard. And many metamaterials researchers are trying to make cloaking a reality, not least because the military has eagerly funded the development of such capabilities.

However, if such applications ever come to pass it will be decades from now. Technologies closer to commercialization are of more interest to Driscoll, a m

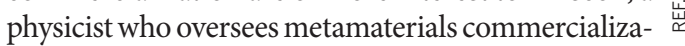
tion at Intellectual Ventures, a patent-aggregation firm in Bellevue, Washington. Applications such as cheaper satellite communications, thinner smartphones and ultrafast optical data processing are "where metamaterials are poised to make a huge impact”, he says.

Researchers still face some daunting challenges, he adds - notably, finding cheap ways to fabricate and manipulate metamaterial elements on a scale of nanometres. But the first metamaterial-based products are expected to come onto the market in a year or so. And, not long after that, Driscoll expects that average consumers will start to enjoy the benefits, such as faster, cheaper Internet connectivity on board planes and from mobile phones. Such applications, he says, will move from being the stuff of 
peoples' fantasies "to becoming things they can't contemplate living without".

The first laboratory demonstration of a metamaterial was announced in 2000 by physicist David Smith and his colleagues at the University of California, San Diego ${ }^{1}$. Following up on theoretical work done in the 1990s by John Pendry of Imperial College London, these researchers showed that an array of tiny copper wires and rings had a negative refractive index for microwaves - meaning that microwave radiation flowing into the material is deflected in a direction opposite to that normally observed (see 'Wave engineering'). That triggered intense interest in metamaterials, in part because the ability to bend radiation in such a way had potential for creating invisibility cloaks.

Since then, Smith and others have explored a host of variations on the metamaterial idea, often looking to manipulate radiation in ways that have nothing to do with a negative refractive index. They have also moved beyond static arrays, devising techniques to change the way the elements are arranged, how they are shaped and how they respond to radiation. The resulting materials can do things such as turn from opaque to transparent or from red to blue - all at the flick of a switch.

\section{MARKET MOVERS}

In January, Smith, now at Duke University in Durham, North Carolina, took on a concurrent role as director of metamaterials commercialization efforts at Intellectual Ventures. "I felt that the time was right, and we didn't need to do any more science for some of these things," he says.

A test case may come as early as next year. Kymeta of Redmond, Washington, a spin-off from Intellectual Ventures, hopes to market a compact antenna that would be one of the first consumer-oriented products based on metamaterials. The relatively inexpensive device would carry broadband satellite communications to and from planes, trains, ships, cars and any other platform required to function in remote locations far from mobile networks.

At the heart of the antenna - the details of which are confidential - is a flat circuit board containing thousands of electronic metamaterial elements, each of which can have its properties changed in an instant by the device's internal software. This allows the antenna to track a satellite across the sky without having to maintain a specific orientation towards it, the way a standard dish antenna does. Instead, the antenna remains still while the software constantly adjusts the electrical properties of each individual metamaterial element. When this is done correctly, waves emitted from the elements will reinforce one another and propagate skywards only in the direction of the satellite; waves emitted in any other direction will cancel one another out and go nowhere. At the same time - and for much the same reason - the array will most readily pick up signals if they are coming from the satellite.

This technology is more compact than alternatives such as dish antennas, says Smith. It offers "significant savings in terms of cost, weight and power draw". Kymeta has already performed demonstrations of this technology for investors and potential development partners. But Smith cautions that the company has yet to set a price for the antenna and that it must still work to bring production costs down while maintaining the strict performance standards that regulatory agencies demand for any device communicating with satellites.

Kymeta has shared so few details of its antenna that researchers say it is hard to offer an evaluation. But Smith is highly regarded in the field. If Kymeta brings the product to market, it may first offer its antenna for use on private jets and passenger planes. If buyers respond well, the company hopes to incorporate the technology into other product lines, such as portable, energy-efficient satellitecommunication units for rescue workers or researchers in the field.

In January, Smith's group turned heads when it announced its demonstration of another metamaterial device: a camera that can create compressed microwave images without a lens or any moving parts ${ }^{2}$. One important application of the device might be to reduce the cost and complexity of airport security scanners.

In their current form, these scanners have to physically sweep a microwave sensor over and around the subject. This produces an unwieldy amount of data that has to be stored before it is processed into an image. The Duke group's device requires very little data storage. It takes numerous snapshots by sending beams of microwaves of multiple wavelengths across the target at about ten times per second. When the microwaves are reflected back by the subject, they fall on a thin strip of square copper metamaterial elements, each of which can be tuned to block or let through reflected radiation. The resulting pattern of opaque and transparent elements can be varied very rapidly, with each configuration transmitting a simplified snapshot of a scanned object into a single sensor. The sensor measures the total intensity of radiation from each snapshot, then outputs a stream of numbers that can be digitally processed to reconstruct a highly compressed image of the subject.

This is admittedly just a first step: demonstrations carried out so far have been crude affairs restricted to two-dimensional images of simple metallic objects. Expanding it to three-dimensional images of complex objects remains a challenge. But if that challenge can be overcome, says Driscoll, airports could retire the bulky, expensive, slow booths that currently constitute security checkpoints, and instead use a larger number of thin, inexpensive metamaterial cameras hooked up to computers. Such a shift, Driscoll says, could extend security scanning to rooms, hallways, and corridors throughout airports and other sensitive facilities.
Wave

\section{engineering}

Metamaterial elements scatter incoming radiation in very precise ways. They can be any shape; common examples include spheres, rings, crosses and chevrons. Their electromagnetic properties can often be changed by software.

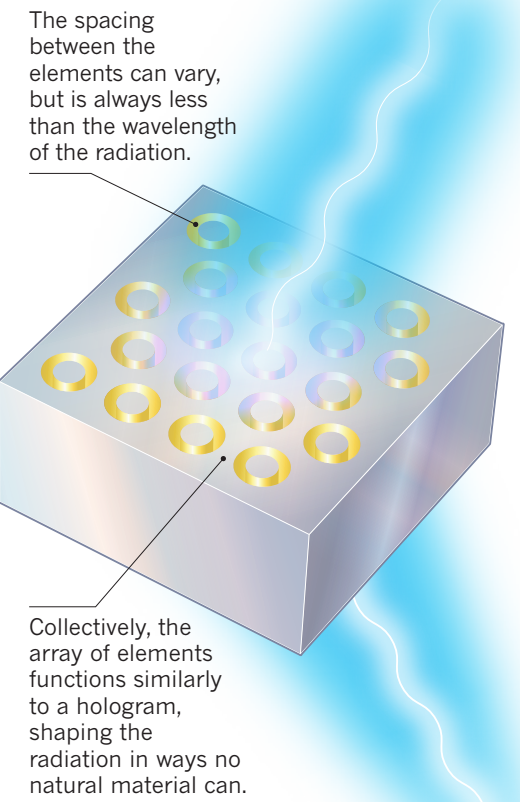

Example:

Negative index

of refraction

Metamaterials can be engineered to bend

radiation in a direction opposite to that observed in ordinary materials.

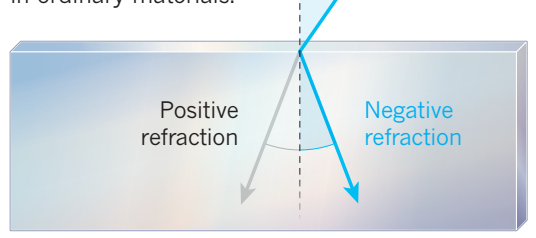

\section{Application:}

Invisibility cloak

A cloak made of a negativeindex metamaterial can bend radiation around an object inside it, making that object seem invisible.

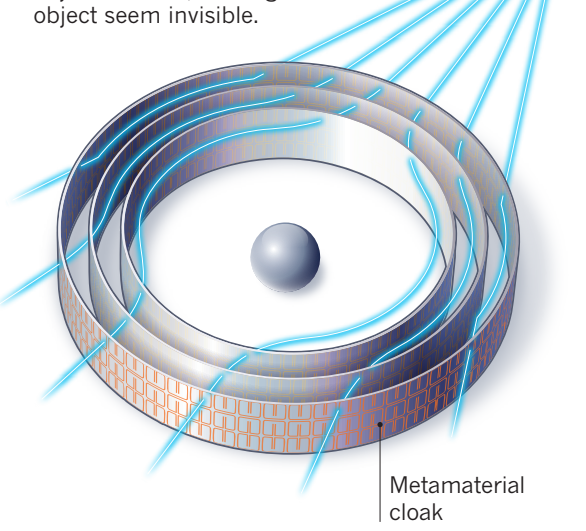


In the meantime, a key research goal for Smith and his group is the development of robust and marketable metamaterial devices that are not restricted to radio, microwave or infrared wavelengths. If the technologies could be made to work with visible light, they would become much more useful for applications such as fibre-optic communications or consumer-oriented cameras and displays.

"It won't be easy," cautions Stephane Larouche, a member of Smith's research team at Duke. For any given type of radiation, he explains, metamaterials can wield their exotic powers only if the elements are smaller and more closely spaced than the wavelength of that radiation. "So the shorter and shorter the wavelength we wish to use, the smaller each metamaterial element must be," says Larouche.

In the microwave and radio regions of the spectrum, this is relatively easy: wavelengths are measured in centimetres to metres. But an optical metamaterial's elements would have to measure considerably less than a micrometre. That is not impossible: today's highperformance microchips contain features only a few tens of nanometres across. But unlike those essentially static features, says Larouche, the metamaterial elements in many applications would need to incorporate ways for software to change their properties dynamically as needed. "Too often we have gorgeous ideas," he says, "but we have no way of fabricating them."

\section{FLAT FOCUS}

Despite these difficulties, workable designs for optical metamaterials have begun to emerge. One was published in $\mathrm{March}^{3}$ by a group working under Nikolay Zheludev, a physicist at the University of Southampton, UK, who directs a research centre focused on metamaterials at Nanyang Technological University in Singapore. The team's device can greatly alter its ability to transmit or reflect optical wavelengths by means of nanometre-scale, electrically controlled metamaterial elements etched from gold film; it could one day serve as a switch in high-speed fibre-optic communications networks.

Meanwhile, because it is so hard to make and control three-dimensional metamaterial arrays at optical scales, some researchers are focusing on two-dimensional 'metasurfaces'. In August 2012, a group led by Federico Capasso at Harvard University in Cambridge, Massachusetts, unveiled a flat metamaterial lens that can focus infrared light to a point in much the same way as a glass lens ${ }^{4}$. "I don't want to claim absolute novelty in this," Capasso says, "but I believe we are the first group to so clearly put flat optics on the agenda for commercial applications."

A conventional lens relies on refraction to bend light to a point by passing it through varying thicknesses of glass. Capasso's lens passes light through a two-dimensional array of gold metamaterial elements carved out of a 60-nanometre-thick silicon wafer using electron-beam lithography techniques developed for the microchip industry. The elements are fixed, so cannot be tuned after fabrication. But by selecting a specific size and spacing during the manufacturing process, physicists can shape light of a chosen wavelength in exactly the right way to make it come to a point.

Capasso warns that commercial applications of such flat lenses are probably still a decade away. This is partly because silicon is a rigid and fragile substrate for etching the elements; researchers are looking at more robust and flexible alternatives that would be easier to handle on the production line. They are also looking for better ways to control the carving of the nanoscale elements, which has to be done very precisely.

But once the technology is mastered, says Capasso, one obvious application is in smartphone cameras. Lenses, along with batteries, are among the most stubborn limiting factors

The team has since been working to refine the superlens concept; in 2007 the researchers advanced the idea by developing 'hyperlenses' from curved, nested layers of compounds such as silver, aluminium and quartz ${ }^{6}$. The lenses not only capture evanescent waves, but can also feed them into a conventional optical system. Ultimately, this could allow sub-wavelength details to be viewed through the eyepiece of a standard microscope. But the complex structure and behaviour of hyperlenses makes them difficult to manufacture and use in this way.

\section{REVERSIBLE FOCUS}

By pairing conventional optics with superlenses and hyperlenses based on metamaterials, Zhang hopes eventually to find applications far beyond microscopy. Just as these constructs can magnify sub-wavelength detail, they can also be run in reverse, directing beams of light into sub-wavelength focal points - a property of potentially revolutionary importance for fabricating minuscule structures using photolithography. If superlenses and hyperlenses can be harnessed for this purpose, the ultra-fine beams of light could be used to etch much smaller features than is possible today. This could greatly increase the density of data

in smartphone thickness, he says, speculating that a smartphone incorporating a flat camera lens could potentially be made "as thin as a credit card". The flat lens also avoids aberrations that plague glass lenses, such as the coloured 'fringes' created by the inability to focus all wavelengths to the same point. This means that Capasso's flat lens could also be used to make better, aberration-free microscopes.

As good as they might ultimately be, the flat lenses would still be subject to the diffraction limit, which dictates that no conventional lens can resolve details much smaller than the wavelength of the light that illuminates its target. This limit averages about 200 nanometres for visible light. But metamaterials offer a means of fabricating 'superlenses' that could surpass such limits, allowing researchers to see sub-wavelength details of target objects such as viruses or the ever-changing structures in living cells.

The key is to recognize that the missing details are still there, carried in 'evanescent' waves of reflected light that die away very rapidly with distance from the illuminated object. Normally, these waves have effectively vanished before they can be captured and focused by a lens. But a metamaterial superlens designed to be placed within tens of nanometres of an object can pick up and magnify these waves.

An early proof-of-concept superlens was demonstrated in 2005 by a group working under Xiang Zhang, a physicist at the University of California, Berkeley ${ }^{5}$. Zhang's group produced a simple metamaterial consisting of a 35-nanometre-thick layer of silver in a sandwich with nanoscale layers of chromium and plastic. storage on optical drives, as well as the number of components that can be crammed onto computer chips.

Smith is cautious on that score, pointing out that hyperlenses and superlenses tend to dissipate substantially more of the light energy passing through them than other advanced lithographic techniques now in development. This, he says, makes them prime examples of "strong and compelling science that is not yet practical for any sort of product path" at optical wavelengths. But, he adds, Zhang's efforts are "heroic experiments that illustrate the potential of metamaterials in a fundamental way".

Zhang concedes that hyperlenses and superlenses are not yet ready for prime time, but believes there is plenty of room for ongoing research to change that situation in the coming years. "The economic impact could be huge," he says. "I am cautiously optimistic that metamaterials, superlenses and lithography will prove truly revolutionary. If people aren't too shortsighted, what we can do with metamaterials will be limited only by our imaginations."

Lee Billings is a freelance writer based in New York.

1. Smith, D. R., Padilla, W. J., Vier, D. C., Nemat-Nasser, S. C. \& Schultz, S. Phys. Rev. Lett. 84, 4184-4187 (2000).

2. Hunt, J. et al. Science 339, 310-313 (2013).

3. Ou, J.-Y., Plum, E. Zhang, J. \& Zheludev, N. I. Nature Nanotechnol. 8, 252-255 (2013).

4. Aieta, F. et al. Nano Lett. 12, 4932-4936 (2012)

5. Fang, N., Lee, H., Sun, C. \& Zhang, X. Science 308 , 534-537 (2005).

6. Liu, Z., Lee, H., Xiong, Y., Sun, C. \& Zhang, X. Science 315,1686 (2007) 